\title{
Overview of LBTI: a multipurpose facility for high spatial resolution observations
}

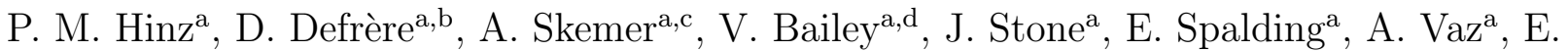 \\ Pinna $^{\mathrm{e}}$, A. Puglisi ${ }^{\mathrm{e}}$, S. Esposito ${ }^{\mathrm{e}}$, M. Montoya ${ }^{\mathrm{a}}$, E. Downey ${ }^{\mathrm{a}}$, J. Leisenring ${ }^{\mathrm{a}}$, O. Durney ${ }^{\mathrm{a}}$, W. \\ Hoffmann $^{\mathrm{a}}$, J. Hill ${ }^{\mathrm{a}}$, R. Millan-Gabet ${ }^{\mathrm{f}}$, B. Mennesson ${ }^{\mathrm{g}}$, W. Danchi ${ }^{\mathrm{h}}$, K. Morzinski ${ }^{\mathrm{a}}$, P. Grenz ${ }^{\mathrm{a}}$, \\ M. Skrutskie ${ }^{\mathrm{i}}$, and S. Ertel ${ }^{\mathrm{a}}$ \\ aSteward Observatory, University of Arizona, 933 North Cherry Ave, Tucson, AZ 85721, USA

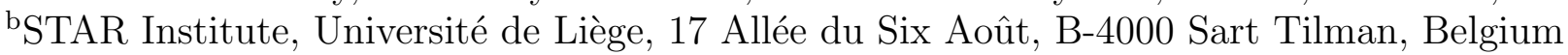 \\ ${ }^{c}$ Department of Astronomy and Astrophysics, University of California, Santa Cruz, 1156 High \\ St, Santa Cruz, CA 95064, USA \\ ${ }^{\mathrm{d}}$ Kavli Institute for Particle Astrophysics and Cosmology, Department of Physics, Stanford \\ University, Stanford, CA, 94305, USA \\ eINAF-Osservatorio Astrofisico di Arcetri, Largo E. Fermi 5, I-50125 Firenze, Italy

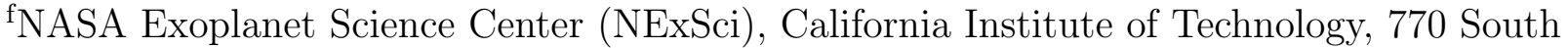 \\ Wilson Avenue, Pasadena CA 91125, USA \\ g.Jet Propulsion Laboratory, California Institute of Technology 4800 Oak Grove Drive, \\ Pasadena CA 91109-8099, USA

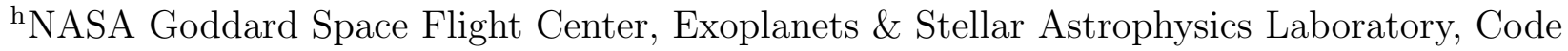 \\ 667, Greenbelt, MD 20771 \\ ${ }^{i}$ University of Virginia, 530 McCormick Rd, Charlottesville, VA, 22904, USA
}

\begin{abstract}
The Large Binocular Telescope Interferometer (LBTI) is a high spatial resolution instrument developed for coherent imaging and nulling interferometry using the $14.4 \mathrm{~m}$ baseline of the $2 \times 8.4 \mathrm{~m}$ LBT. The unique telescope design, comprising of the dual apertures on a common elevation-azimuth mount, enables a broad use of observing modes. The full system is comprised of dual adaptive optics systems, a near-infrared phasing camera, a 1-5 $\mu \mathrm{m}$ camera (called LMIRCam), and an 8-13 $\mu \mathrm{m}$ camera (called NOMIC). The key program for LBTI is the Hunt for Observable Signatures of Terrestrial planetary Systems (HOSTS), a survey using nulling interferometry to constrain the typical brightness from exozodiacal dust around nearby stars. Additional observations focus on the detection and characterization of giant planets in the thermal infrared, high spatial resolution imaging of complex scenes such as Jupiter's moon, Io, planets forming in transition disks, and the structure of active Galactic Nuclei (AGN). Several instrumental upgrades are currently underway to improve and expand the capabilities of LBTI. These include: Improving the performance and limiting magnitude of the parallel adaptive optics systems; quadrupling the field of view of LMIRcam (increasing to 20"x20"); adding an integral field spectrometry mode; and implementing a new algorithm for path length correction that accounts for dispersion due to atmospheric water vapor. We present the current architecture and performance of LBTI, as well as an overview of the upgrades.
\end{abstract}

Keywords: LBT, interferometry, infrared instruments, adaptive optics, imaging

Further author information: (Send correspondence to Phil Hinz)

E-mail: phinz@as.arizona.edu, Telephone: 15206217866

Optical and Infrared Interferometry and Imaging V, edited by Fabien Malbet,

Michelle J. Creech-Eakman, Peter G. Tuthill, Proc. of SPIE Vol. 9907, 990704

(C) 2016 SPIE · CCC code: 0277-786X/16/\$18 - doi: 10.1117/12.2233795

Proc. of SPIE Vol. 9907 990704-1 


\section{INTRODUCTION}

The Large Binocular telescope ${ }^{1}$ (LBT) is a powerful facility that has integrated precision adaptive optics capability and the ability to combine the beams from the $2 \times 8.4 \mathrm{~m}$ apertures on the telescope structure. The LBT Interferometer (LBTI) project ${ }^{2}$ was developed to exploit this unique telescope architecture, producing high spatial resolution observations. One mode in particular that has high priority is the use of LBTI as a nulling interferometer. The technique of nulling interferometry can be used to detect warm dust from debris disks around main sequence stars. Possible future missions designed to image an Earth-size planet around another star will need to contend with the level of warm dust in these systems. Consequently, NASA has supported the development of LBTI, as well as a key survey using the instrument. The LBTI project is thus primarily focused on the Hunt for Observable Signatures of Terrestrial planetary Systems (HOSTS). Under HOSTS, LBTI is used to detect and resolve the thermal emission in the $10 \mu \mathrm{m}$ wavelength region due to zodiacal dust around nearby stars.

Complementary to the nulling interferometry mode, LBTI was also designed to accommodate Fizeau imaging, which harnesses the the full $22.7 \mathrm{~m}$ baseline of the binocular telescope to enable high spatial resolution observations over a large field-of-view.

The platform of LBTI, due to its flexible design, enables additional modes beyond these two core capabilities. This paper summarizes the status and performance of LBTI for its range of current and planned uses.

\section{LBTI ARCHITECTURE}

The LBTI instrument architecture is designed to allow multiple science cameras to make use of the adaptive optics systems and beam combination capabilities. Consequently, the front-end of the system is a reflective beamcombiner that creates an $\mathrm{f} / 15$ envelope of beams at the combined focus in the center of the instrument volume. Visible light from each LBT aperture is diverted via dichroics to pyramid wavefront sensors. Pathlength correction mechanisms are integrated into the beamcombiner. A sketch of the instrument is shown in Figure 1.

The left mirror in the beamcombiner provides fast pathlength correction (FPC) with $70 \mu \mathrm{m}$ of physical stroke via Piezo-electric transducers (PZT), capable of introducing $140 \mu \mathrm{m}$ of optical path difference (OPD) correction. The right mirror provides larger stroke (40 mm of motion), slow pathlength correction (SPC) via a stepper motor-driven translation stage. In practice we use the SPC to scan and acquire interference fringes. The FPC provides the atmospheric corrector mechanism. The bottom of Figure 1 shows how the light is split up once it enters the Nulling and Imaging Cryostat (NIC). A slightly more detailed sketch of the nulling portion of NIC is shown in the right side of Figure 1. The two beams of the telescopes are overlapped on a $50 \%$ transmissive beam-splitter (BS) which can be translated to equalize pathlength. Precise pathlength adjustments can be put in with two PZT devices. Each has a throw of $11 \mu \mathrm{m}$, or $22 \mu \mathrm{m}$ in OPD.

\subsection{The Beamcombiner}

Light from each telescope is directed by the tertiary to a central location on the telescope platform. This location houses the LBTI structure. As the telescope light enters the LBTI area from each side, the visible light is reflected via long-pass dichroics with a wavelength cutoff at $1 \mu \mathrm{m}$. The infrared light is allowed to enter an evacuated beamcombiner area where all optics and radiation baffles are cooled via discrete liquid nitrogen vessels. The telescope foci are just inside the entrance window on each side of the beamcombiner. The infrared light is directed to an off-axis reflective ellipsoid that provides a 2.75:1 magnification ratio. These ellipsoid mirrors create an image of the secondary mirror at two flat "pupil" mirrors. These mirrors can be actuated and adjusted in pathlength to fine-tune the image overlap and pathlength difference between the telescopes. The left pupil mirror is a three-axis PZT-driven assembly that can provide $100 \mu \mathrm{m}$ of pathlength correction when operated at cryogenic temperatures. The right pupil mirror is mounted on a slowly moving translation stage that can provide more than $20 \mathrm{~mm}$ of pathlength adjustment. After reflecting off the pupil mirrors, the light is directed to a central mirror assembly that direct both beams toward the bottom of the beamcombiner. This mirror assembly is referred to as the "roof mirrors" as they resemble an inverted roof. The roof mirrors can be adjusted in tip and tilt to refine the alignment of the beamcombiner optics so that the beams are overlapped and the combined exit pupil mimics the geometry of the telescope entrance pupil. 

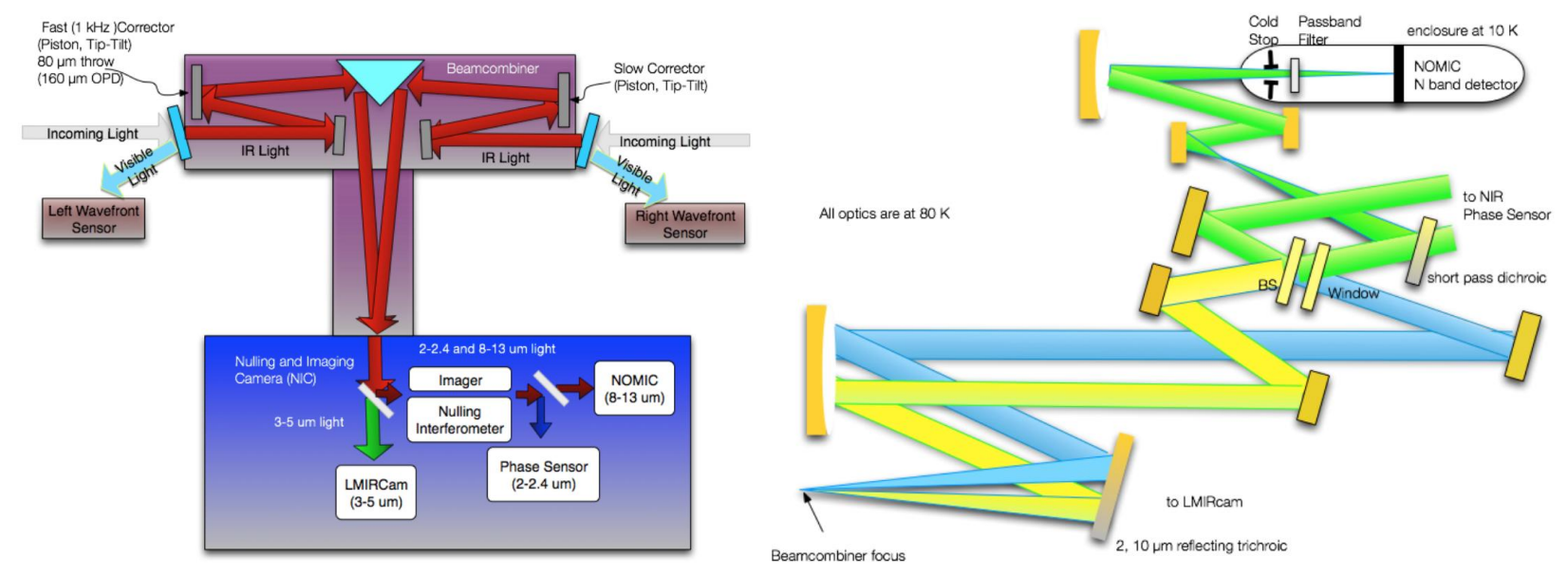

Figure 1. Sketch of the LBTI beam-combiner and its Nulling and Imaging Camera (NIC) (left) and the nulling portion of NIC (right).

The light from the beamcombiner comes to a combined focus approximately $125 \mathrm{~mm}$ inside the entrance window for the Nulling and Imaging Camera (NIC) which houses the L and M band InfraRed Camera (LMIRcam) as well as the Nulling-Optimized Mid-Infrared Camera (NOMIC). Light is split after the focal plane by a trichroic that sends 3-5 $\mu \mathrm{m}$ light to LMIRCam while reflecting 2-2.4 $\mu \mathrm{m}$ light to the phase sensor (PhaseCam) and 8-13 $\mu \mathrm{m}$ light to NOMIC.

\subsection{LMIRCam}

LMIRCam $^{3}$ is a camera designed for imaging at a 1:1 magnification of the combined focus provided by the LBTI beam-combiner. Re-imaging is accomplished via two reflective powered optics. Each powered optic forms an image of the input focal plane with a 1:1 magnification. The shape of each mirror that provides good image quality is a bi-conical surface with slightly different radii and conic constants for the in-plane and out-of-plane coordinates of the optics. The second bi-conical mirror corrects off-axis aberrations put into the first bi-conical mirror.

This optical design creates an intermediate focal plane (useful for slits, integral field unit (IFU) optics or occulting masks) as well as an upstream and downstream pupil plane. Interchangeable cold stops and a filter wheel are placed at the first pupil plane in LMIRCam, while additional filters and a disperser are housed in additional wheels located at the second pupil plane.

\subsection{NOMIC}

NOMIC uses the same dual bi-conical reflective design to form intermediate pupil and focal planes. However, the NOMIC camera also houses a nulling interferometer. This is accomplished by placing selectable diverter optics after the first powered mirror that redirect the light to a nulling interferometer, before reinserting the light into the original beam train. In this way either nulling or imaging interferometry can be accomplished at 8-13 $\mu \mathrm{m}$.

\section{LBTI CONTROL ARCHITECTURE}

LBTI observations are coordinated by the science cameras (NOMIC or LMIRCam) that can be accomplished either via web-based graphical user interfaces, or via a python-based scripting environment. All hardware is controlled via Instrument Neutral Distributed Interface ${ }^{4}$ (INDI) software drivers. As shown in Figure 2, there are three high-speed loops that are operating during a typical LBTI operation, along with slower speed communication that allows for alignment and off-loading. 


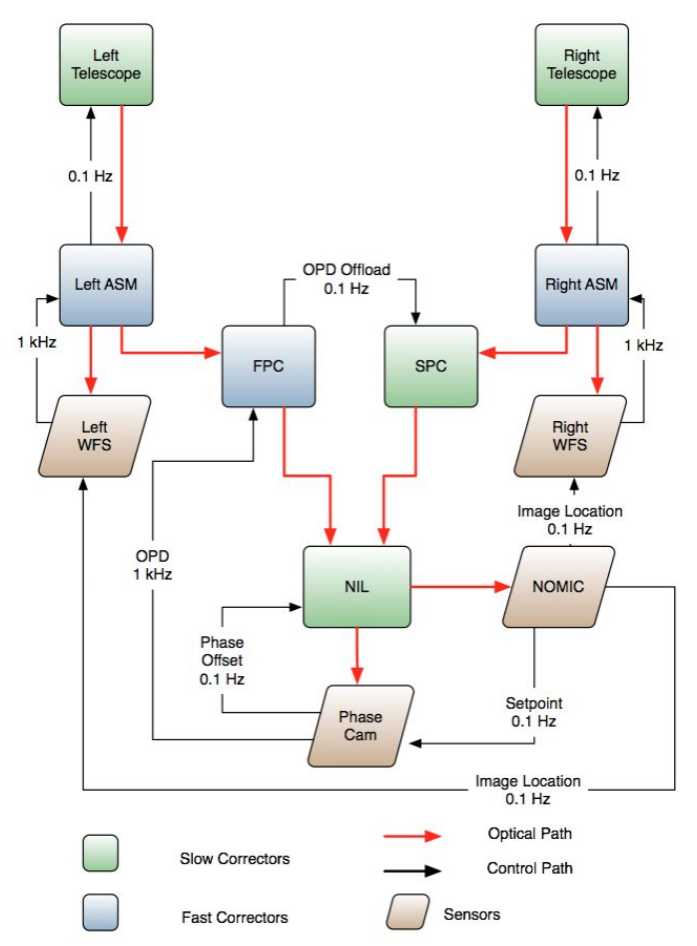

Figure 2. Control architecture for LBTI. Three main control loops are used to deliver a wavefront-corrected and phased beam to the final combined focal plane. Accumulation of errors in tip/tilt and high-order modes for each telescope is sent to the telescope pointing system for correction, while buildup of optical pathlength difference (OPD) errors are compensated for by the beamcombiner slow pathlength corrector (SPC).

\subsection{Adaptive Optics}

The LBTI adaptive optics system uses the facility adaptive secondary mirror for wavefront corrections at rates of up to $1 \mathrm{kHz}$. Wavefront sensing is provided by pyramid-based wavefront sensors (WFS) with up to 30 subapertures across the diameter of each telescope pupil. These systems are clones of the First-Light Adaptive Optics (FLAO) WFS systems ${ }^{56}$ mounted in adjacent ports and developed at Arcetri Observatory. The only significant difference is that the LBTI WFS assemblies are mounted in a fixed orientation relative to the telescope pupil and thus do not have rerotator optics that are part of the FLAO WFS.

\subsection{PhaseCam}

The phase sensing for LBTI is carried out via the nulling interferometry outputs. Both outputs are sent to a Near-Infrared PICNIC sensor, where two quadrants are used to sense the two outputs at $1 \mathrm{kHz}$. Various optics can be put into the beam that create a large ( 100 pixel) pupil image, a small ( $\sim 15$ pixel) pupil image, an image of the star, or a low resolution spectrum of the star.

The sensing approach, first developed in Fall $2012,{ }^{7}$ was to use the small pupil images. It was found that, due to the lateral image dispersion between $2.2 \mu \mathrm{m}$ and $11 \mu \mathrm{m}$ in the beamsplitter, a well overlapped set of images at $11 \mu \mathrm{m}$ corresponds to a tilt difference of roughly 3 fringes across the pupil at $2.2 \mu \mathrm{m}$. The resulting pupil image was a uniform intensity circle that had three vertical interference fringes across it. This allowed extraction of separate tip-tilt and phase variations via a Fourier transform of the detected light. Figure 3 shows an example of how this is carried out. The Fourier transform of the pupil image provides peaks in the resulting image, and this approach has the nice feature of providing a signal in the Fourier plane well separated from the zero-frequency component. The location of the peak in the amplitude plane corresponds to the tip/tilt error signal. The argument of the complex number at the peak location is a measurement of the path length error. 

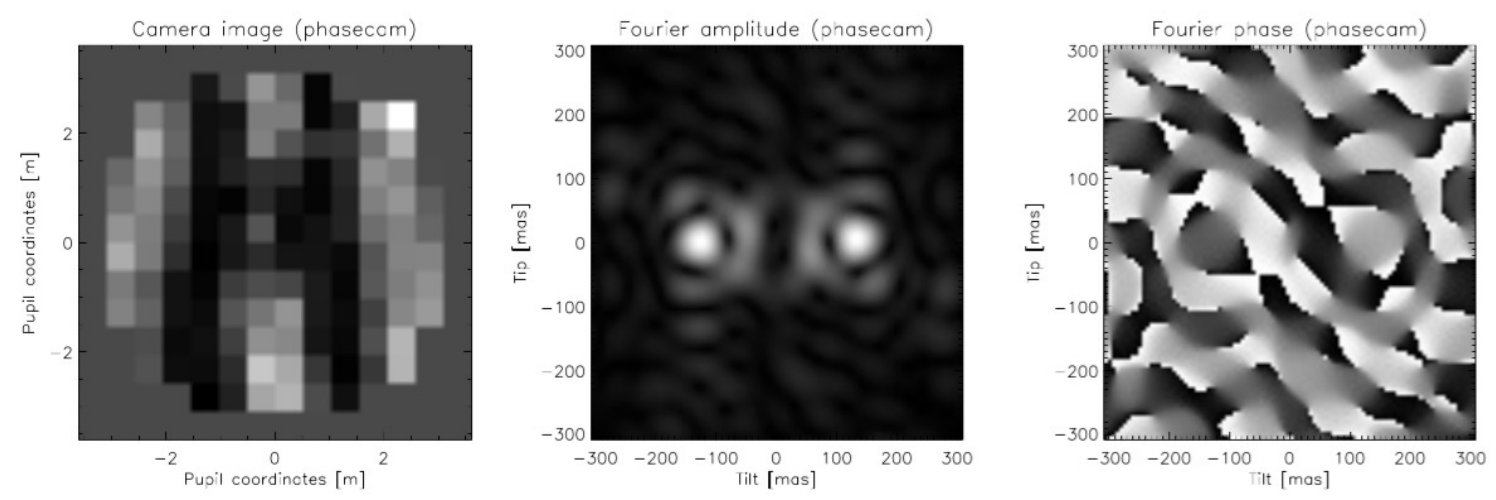

Figure 3. An example of the initial camera image of the interference at $\mathrm{K}$ band (left), the amplitude of the Fourier transform of the image (center), and the phase of the Fourier transform (right). The center and right images are used as the error input for controlling pathlength variations.

Although both outputs can be used (and difference) to provide a signal less susceptible to intensity variations, we found in practice that the use of one output is sufficient to control the phase. In typical operation, these measurements are made at $1 \mathrm{kHz}$. The phase is unwrapped, and this error signal is then fed to an integral error control loop.

A more recent addition to the phase sensor has been to measure the second output at $1.65 \mu \mathrm{m}$, rather than $2.2 \mu \mathrm{m}$. This has the advantage of measuring phase dispersion between the beams. Such a signal is useful for measuring (and correcting for) water vapor variations between the beams. A separate paper by Defrère et al. in this proceeding discusses the status of this improvement in more detail.

In spring 2016, we also began integrating accelerometer-based estimates of the optical pathlength difference (OPD) variations due to vibrations of the telescope structure. We successfully demonstrated the use of the OPD and Vibration Monitoring System (OVMS) system to reduce the OPD RMS error from $450 \mathrm{~nm}$ to approximately $300 \mathrm{~nm}$, primarily due to better suppression of a $12 \mathrm{~Hz}$ telescope vibration. A separate paper by Boehm et al. in these proceedings describes the formulation and implementation of OVMS+ in more detail.

\section{OBSERVING MODES}

The LBTI facility can be used in many different modes of observations, from single aperture observations to phased dual aperture observations. We describe below the state of use for these several modes.

\subsection{Adaptive Optics Imaging}

The alignment optics of LBTI allow for differential pointing of each side by roughly 10 arcseconds on the detector array. This allows for easy adjustment of the beams to either overlap them on one of the science detectors, or to place the images side-by-side. Further, the cameras have internal cold stops that can allow for blocking of either the left or right telescope, along with the background associated with the telescope.

This flexibility allows for mix-and-match styles of observations. For programs that require efficiency, short exposures, and many telescope pointings, we have found it simplest to use only one side of the telescope. For programs that are contrast-limited, we have found it more useful to acquire dual AO images with the images separated by half the detector width. Low signal-to-noise companion data can either be confirmed or ruled out with the simultaneous images from each telescope. This style of operation can also allow for different science detectors to be used on each side of the telescope. For example, it is possible to acquire NOMIC images with the left side while acquiring LMIRCam images with the right side, with independent nodding throws and timing for each camera. Figure 4 shows two examples of imaging carried out with LMIRCam, exploring the structure of debris disks ${ }^{8}$ and placing constraints on inner companions to a known multiple-planet system. ${ }^{9}$ 

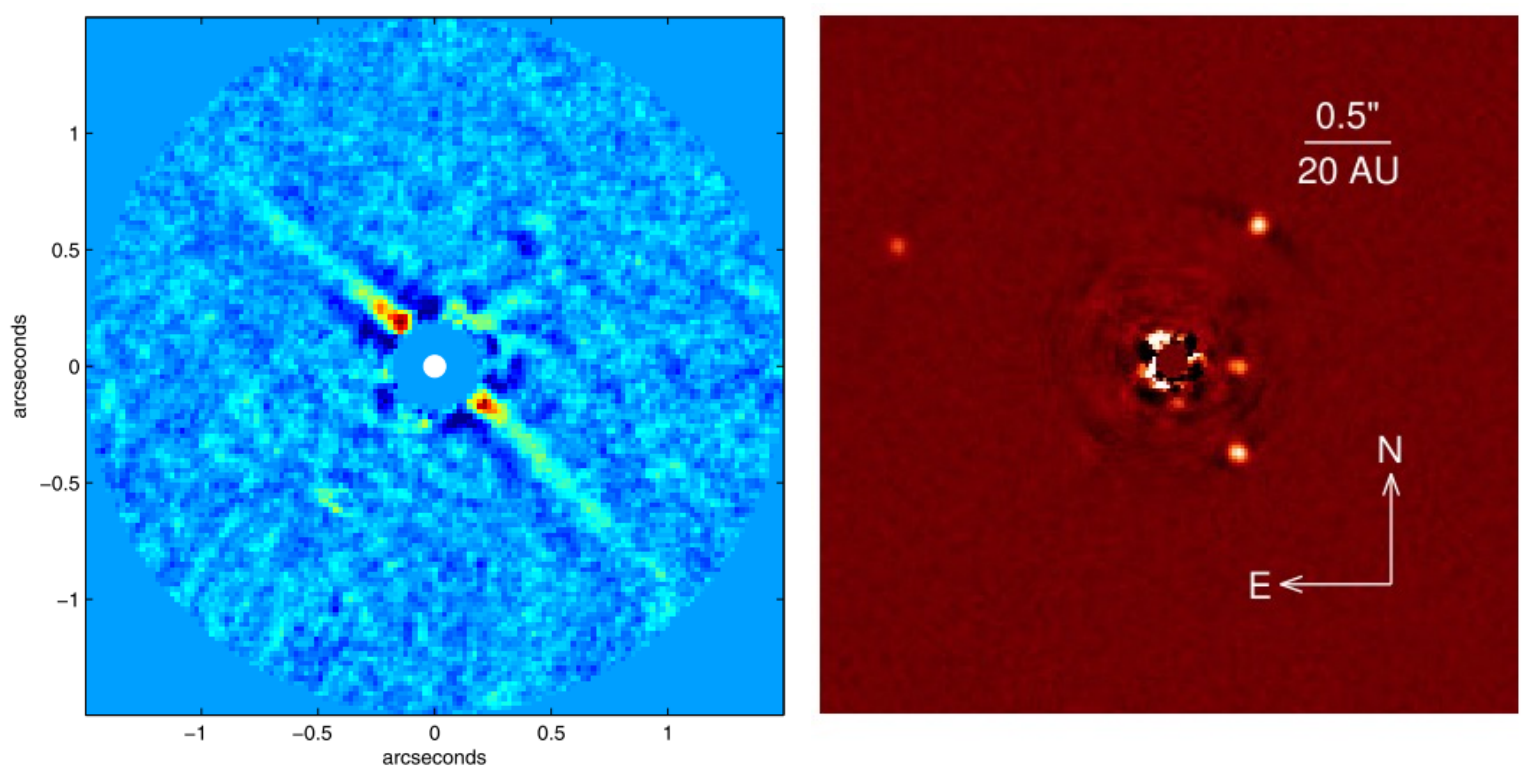

Figure 4. Example images of adaptive optics imaging carried out with LBTI. The left image shows the edge-on debris disk around HD $15115 .{ }^{8}$ The right image shows the planetary system around HR $8799 .{ }^{9}$ Both observations were obtained in the $\mathrm{L}^{\prime}$ passband.

A variation on this flexibility has been explored by Spalding et al. as described in a separate paper in these proceedings to acquire precise photometry via observations of a science target with one telescope and a nearby ( $\lesssim 2$ arcminutes) reference star with the other telescope. This has the potential for acquiring improved photometry at longer wavelength where the detector FOV and sky background often limit the availability of a reference star that can be measured simultaneously.

\subsection{Nulling Interferometry}

Nulling interferometry is implemented on the LBTI by sending each telescope beam through opposite sides of a single-pass $50 \%$ transmissive beam-splitter at near normal incidence. The null is made achromatic by creating a slight difference in thickness of zinc selenide (the beam-splitter and compensating window substrate) between the two beams. Fine alignment of the beams can be accomplished by adjustment of two three-axis PZT tip-tilt-piston platforms, as shown in the right sketch of Figure 1.

The interferometer is tuned to null by adjusting the phase sensor setpoint, while measuring the flux at 11 $\mu \mathrm{m}$. This process has been scripted as part of the setup procedure for nulling observations.

Observations using nulling interferometry are typically carried out by acquiring many $(\sim 1000)$ short $(\sim 50$ ms) exposure images per pointing. The variations in the intensity can be used to separate the intrinsic null depth from a nonzero null due to phase variations in the system by fitting the histogram ${ }^{10}$ of these many null measurements as shown in Figure 5. Mennesson et al. in these proceedings describe the use of this Nulling Self-Calibration (NSC) technique for the LBTI nulling observations.

\subsection{Coherent Imaging}

The LBTI beam combination provides the ability to form images that are limited by diffraction from the 22.7 $\mathrm{m}$ edge-to-edge spacing of the combined LBT apertures. By equalizing the pathlength between the apertures, fringes in the direction of the baseline can be seen for any point-like object in the field-of-view. Figure 6 shows these raw images for observations of the moon Io. ${ }^{11}$ As the LBTI tracked the moon during transit, the baseline for the fringes changed relative to the sky orientation. These sequences of images allows deconvolution to generate an image of the object that can provide much better discernment of fine features, as shown in Figure 7. 

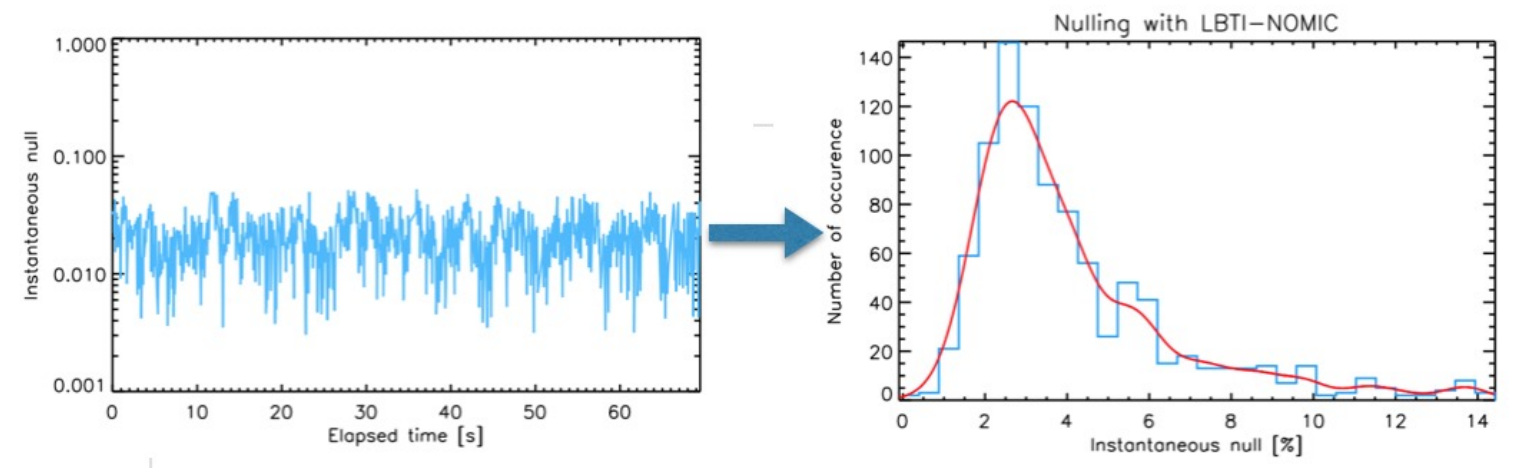

Figure 5. Plot of a time series of null measurements, from Defrère et al. ${ }^{10}$ (left). The resulting histogram (right) is fit to extract the astrophysical null, distingishing it from null variations due to vibrations and wavefront fluctuations.

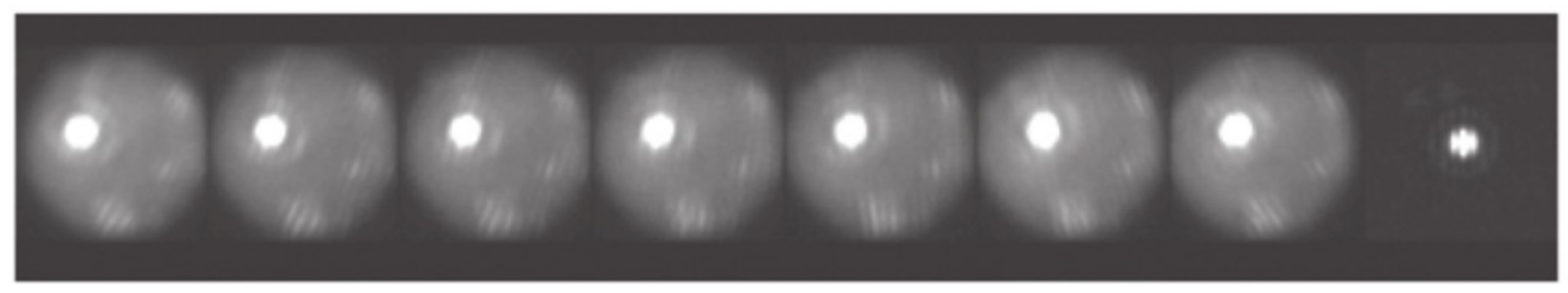

Figure 6. Raw Images from Conrad et al. ${ }^{11}$ of the moon Io in LBTI coherent imaging.

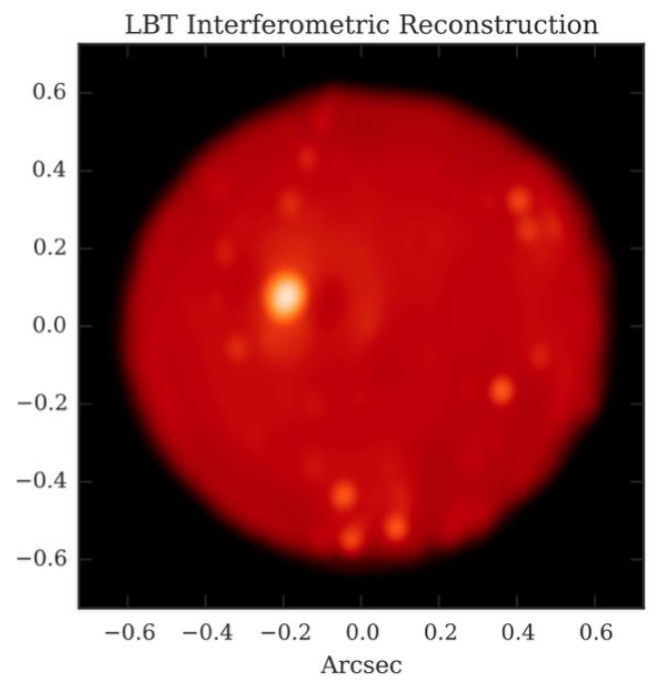

Figure 7. Resulting image reconstruction achieved of Jupiter's moon Io, using the images in Figure 6. 


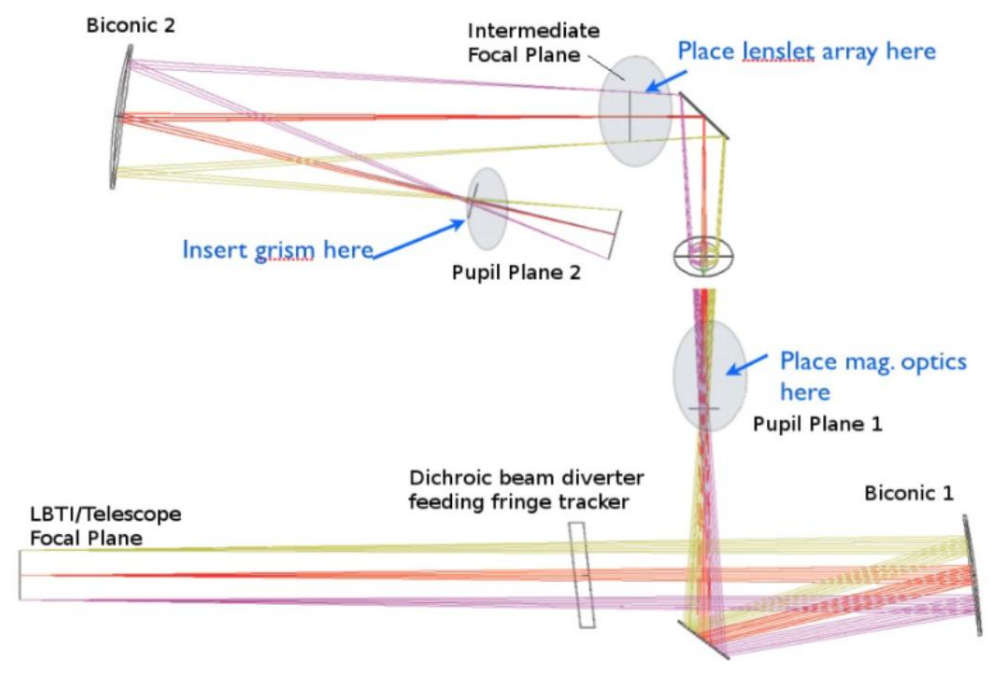

Figure 8. Optical path and location of the IFU optics for ALES.

\subsection{Non-Redundant Mask Observations}

Both LMIRCam and NOMIC have masks in their cold stop wheels that allow for non-redundant mask observations to be carried out. For each camera there are 12 and 24 hole masks, as well as partial annular rings. Currently, the NRM has been used with LMIRCam to track the orbit of LkCa 15 at L' band using dual intraaperture NRM measurements. ${ }^{12}$ Inter-aperture measurements will require integration of this technique with phase stabilization. This is possible, but has, to-date, been limited by the ability of the phasing system to work at fainter magnitudes.

\subsection{Integral Field Spectroscopy}

LMIRCam has recently been augmented with the Arizona Lenslets for Exoplanet Spectroscopy (ALES), an integral field spectrometer design. ${ }^{13}$ To accomplish this, a set of magnifier optics is placed just after the first pupil, creating a magnified image at the intermediate focus. A lenslet array is placed at the the focal plane, followed by a direct-vision dispersing prism at the second pupil plane, as shown in Figure 8. The resulting additional optics enables the use of LMIRCam for low resolution spectroscopy over a 1.3 arcsecond field of view. Figure 9 shows an initial use of ALES to observe Jupiter's moon, Io. As of summer 2016, the initial magnification $(8.2 \times)$ has been tested. An additional set of magnifiers and dispersers will be added to broaden the range of capabilities for ALES in 2017.

\section{CURRENT PERFORMANCE}

The capabilities of LBTI have been characterized for several of the observing modes described above. Here we summarize typical observing capabilities for the more established modes, as reported by LBTI publications to-date.

\subsection{Imaging Sensitivity Limits}

Imaging sensitivity limits, extracted from actual observations with LBTI at various wavelengths are shown in Table 1. While not a complete list of sensitivities, these illustrate the achieved performance of the instrument at various wavelengths in routine use.

\subsection{High Contrast Observations}

Estimates of sensitivity vs. separation for faint companion detection has been measured for LBTI/LMIRCam for several different stars under a range of conditions. Two useful contrast curves are shown in Figure 10 for typical exoplanet survey observations ${ }^{14}{ }^{9}$ 


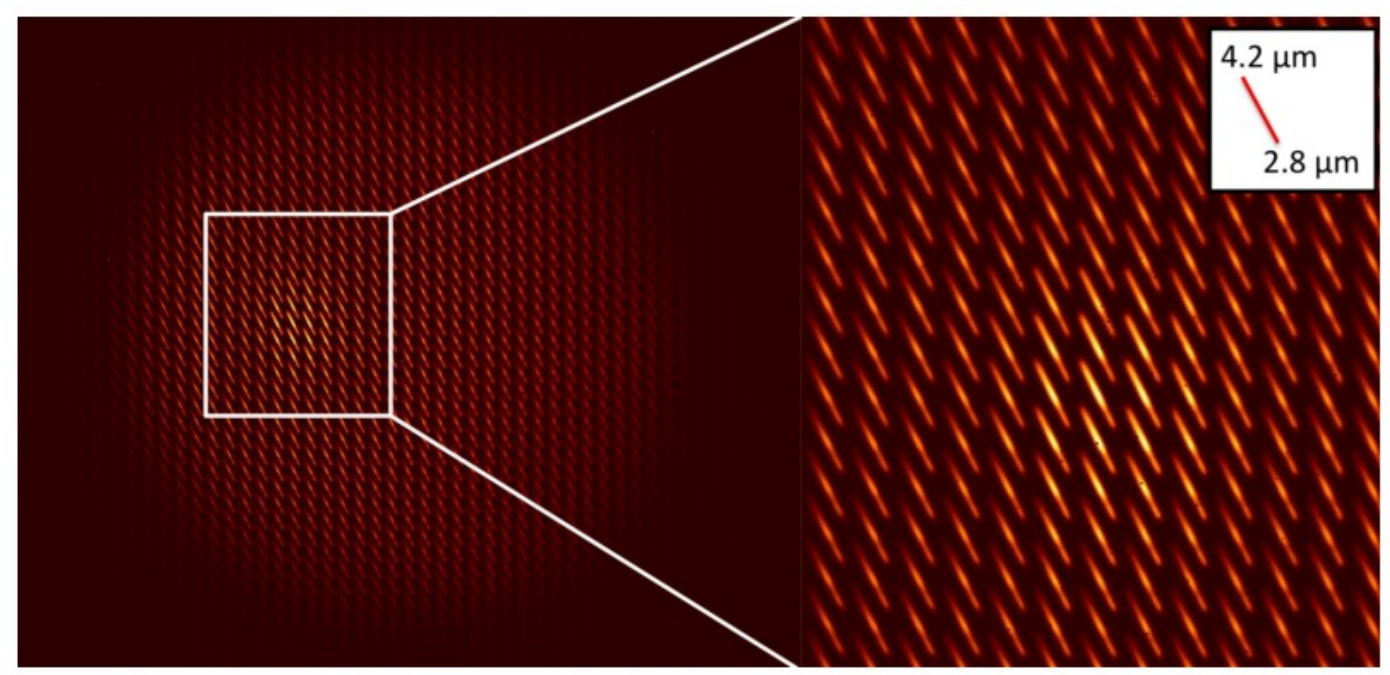

Figure 9. Observation of Jupiter's moon, Io, with ALES. Each spaxel is dispersed to create a low resolution spectrum that spans $2.8-4.2 \mu \mathrm{m}$. A bright volcano can be seen in the raw data.
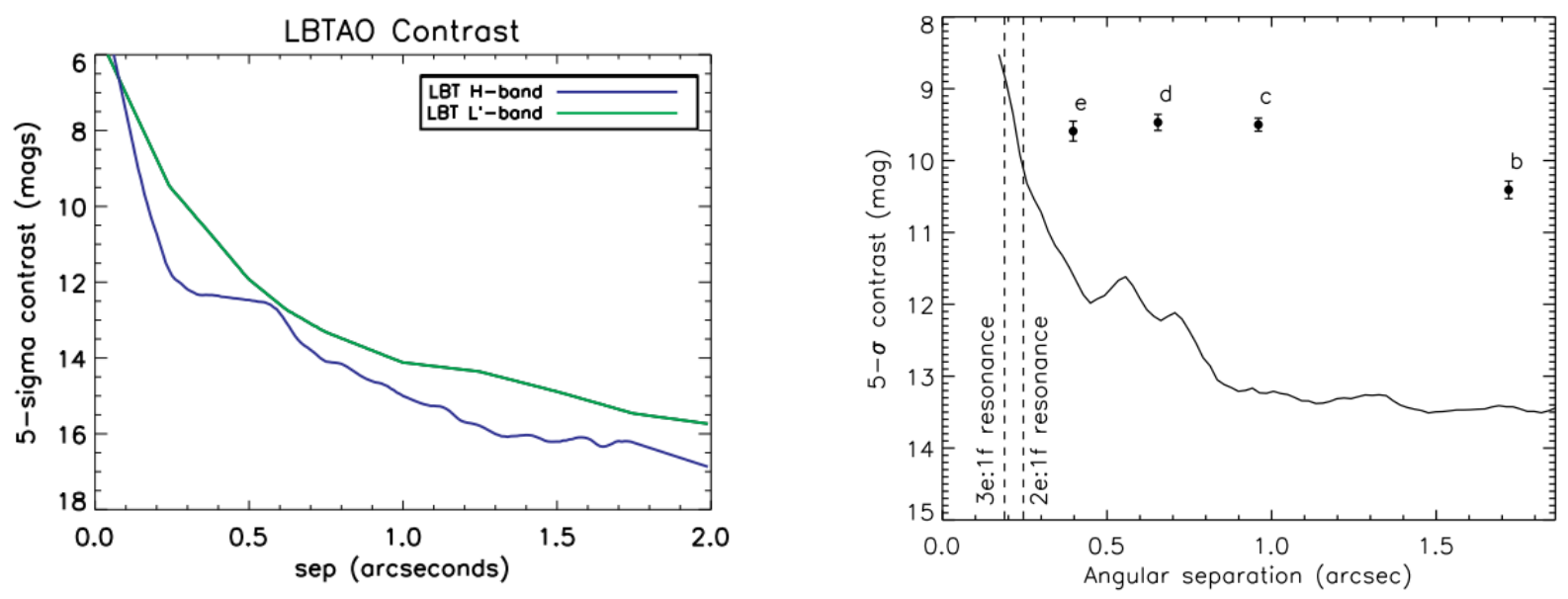

Figure 10. Estimated contrast limits for LMIRCam at L' with Vega ${ }^{14}$ (left) and HR $8799 .^{9}$ Note that for HR8799 the limiting magnitude is dominated by sky noise beyond $\sim 0.8$ arcsec. 
Table 1. LBTI Sensitivity Limits from published results. Quoted limits are $5 \sigma$ detections.

\begin{tabular}{|l|l|l|l|l|}
\hline Band & Time (hrs) & Limit (Vega mag.) & Comment & Reference \\
\hline 3.71 & 1 & 17.6 & dual & Skemer et al. 2014 ${ }^{14}$ \\
\hline L' & 3.0 & 19.1 & one side & Rodigas et al. 2014 \\
L' & 1.75 & 18.7 & one side & Maire et al. 2015 \\
L' & 0.5 & 16.0 & one side & Matthews et al. 2014 \\
\hline M' & 0.74 & $14.8-15.5$ & one side & Bonnefoy et al. 2014 \\
\hline N' & 0.16 & 12.5 & nulling & Defrère et al. 2015 \\
\hline
\end{tabular}

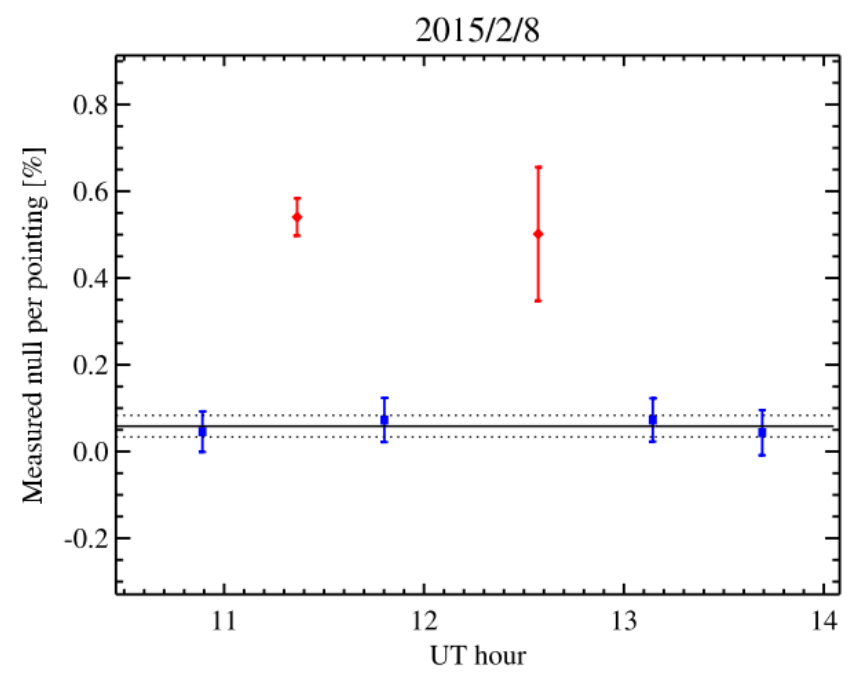

Figure 11. Null depth measurements per pointing as a function of UT time for $\beta$ Leo (red diamonds) and its calibrators (blue squares, data from February 8, 2015). The estimated instrumental null floor is represented by the solid black line and the corresponding 1- $\sigma$ uncertainty by the dotted lines.

\subsection{Nulling Interferometry}

For nulling interferometry, the scientific motivation is to detect or set limits on warm, zodiacal dust around nearby stars. We expect a zodiacal dust disk ${ }^{18}$ around a solar twin to emit $5 \times 10^{-5}(50 \mathrm{ppm})$ of the stellar flux at $11 \mu \mathrm{m}$. The LBTI setup will receive $2 / 3$ of the disk flux at its final focal plane (33 ppm), if the star is at 10 pc distance. The goal of LBTI is to detect disks that are significantly brighter than our own, and that might affect the detection performance of a future exo-Earth imaging mission.

Commissioning of nulling interferometry was carried out in 2014-15. As more routine and repeatable observing sequences the nulling uncertainty was gradually improved from $\sim 2000 \mathrm{ppm}$ to $400 \mathrm{ppm}$ in early 2015 . A few HOSTS sample stars have been observed, but the bulk of the survey ${ }^{19}$ is expected to be carried out in 2016-2018.

Figure 11 shows an example of a HOSTS observation. The blue observations are an estimate of the null level from calibrator stars. The red points show the measured null on the nearby A star $\beta$ Leo. The estimated uncertainty for this complete sequence of 7 pointing is approximately $500 \mathrm{ppm}$. Scaling this to the 9 pointings expected for a typical HOSTS observing sequence, the current null uncertainty is estimated to be $400 \mathrm{ppm}$. This corresponds to 12 zodi for the solar twin at $10 \mathrm{pc}$ used as a HOSTS metric. For the specific parameters of $\beta$ Leo this corresponds to a limit of 8 zodi. The detection, shown in Figure 11, is equivalent to a 90 zodi dust disk. 


\section{PLANNED IMPROVEMENTS}

Although the LBTI is routinely used in the range of observing modes above, it is also an evolving instrument with several planned improvements over the next several years.

\subsection{AO Improvements - SOUL}

The current WFS detector is limited by the read noise inherent in devices when LBTI was deployed, and does not fully exploit the number of modes that could be applied to the 672 actuator adaptive secondary mirrors. A planned upgrade, the Single conjugate adaptive Optics Upgrade for the LBT (SOUL), described by Pinna et al. in these proceedings, will replace this detector with an electron-multiplied CCD (EMCCD) that can measure 40 subapertures across the diameter of each telescope. This has the potential to improve the bright star AO performance, as well as extend the faint limit of guide stars by a magnitude or more.

\subsection{LMIRCam Larger Field-of-View and Integral Field Spectroscopy}

The ALES upgrade to LMIRcam implements a single magnification $(8.2 \times)$ and wavelength range $(2.9-4.1 \mu \mathrm{m})$ using a transmissive magnifier lens system. Further, off-axis astigmatism from the LMIRcam biconic, limits offaxis image quality. In addition, the current H2RG detector is only used over a 1024x1024 sub-frame currently, due to a limitation in electronics originally developed for a previous generation array.

In summer 2016, we plan to replace the existing electronics with a Teledyne SIDECAR system. This will expand the field-of-view to 20 arcsec. The LMIRCam optics were designed to accommodate this larger field-ofview already.

Additional magnifier optics and dispersion ranges will be implemented over the next couple years to broaden the use of ALES. Specifically, we plan to fabricate reflective magnifiers with $4.1 \times, 8.2 \times, 16.5 \times$ and $33 \times$ magnification. The reflective magnifier design will enable use of the IFU from 1.5-5 $\mu \mathrm{m}$. We have planned additional disperser modes that range from $\mathrm{R}=20$ to $\mathrm{R}=150$ to address detection of features such as $\mathrm{H}_{2}$ and $\mathrm{PAH}$.

\subsection{Shorter Wavelength Interferometry}

The current LMIRcam optics provide nyquist sampling for the coherent imaging PSF as short as $\sim 2 \mu \mathrm{m}$. The magnification optics, developed for ALES, can also enable interferometry at wavelengths as short as $1 \mu \mathrm{m}$. Specifically, the $4.1 \times$ magnifier provides a field-of-view of 5 arcsec, sufficient to observe many objects of interest for high-spatial resolution, while sampling the interferometric PSF with pixels spanning 2.5 milliarcseconds each. This is well above Nyquist sampling limits for all NIR observations.

An additional change in a dichroic in NIC is needed to pass light shorter than $1.2 \mu \mathrm{m}$.

\subsection{SHARK}

The System for coronography with High-order Adaptive optics from R to K band (SHARK), are two planned instruments, one to be fed by each LBT aperture in the NIR and visible wavelength regions. The instruments are described by Farinato et al. and by Pedichini et al. in these proceedings. They will leverage the LBTI WFS and software infrastructure to broaden the wavelength coverage of LBT observations carried out at high contrast. The SHARK cameras are planned for mounting on the LBTI structures near the bent Gregorian focus of each telescope. Pickoff mirrors for each camera will be inserted that allow them to use the WFS infrastructure for LBTI.

In addition to science programs that use these instruments, the LBTI infrastructure will benefit from additional cameras that allow sequential or even simultaneous acquisition of data sets probing $0.6-13 \mu \mathrm{m}$ in wavelength. 


\section{5 iLOCATER}

The precision AO-fed spectrometer, iLOCATER, is planned for integration into the LBTI infrastructure. The LBTI beam-combiner provides flexible beam steering and fore-optics for this novel instrumentation.

Initial tests of the iLOCATER single-mode fiber coupling were carried out with the LBTI in Spring 2016, as described by Bechter et al. in these proceedings. A coupling efficiency $>20 \%$ was accomplished, validating the approach of using a single-mode fiber, and a high-perfomance AO system to greatly simplify the design of a precision radial-velocity spectrometer.

\section{SUMMARY}

The LBT Interferometer has become a platform for a broad range of observations using high spatial resolution techniques. We anticipate a focus on the HOSTS survey over the next two years in particular. Additional efforts are gradually being carried out to use coherent imaging and nonredundant masking to realize the benefits of the $22.6 \mathrm{~m}$ LBTI resolution. New modes now in the process of being implemented or planned will broaden the use of LBTI to provide a versatile tool for carrying out high-spatial-resolution observations.

\section{ACKNOWLEDGMENTS}

LBTI is funded by a NASA grant in support of the Exoplanet Exploration Program. The LBT is an international collaboration among institutions in the United States, Italy and Germany. LBT Corporation partners are: The University of Arizona on behalf of the Arizona university system; Istituto Nazionale di Astrofisica, Italy; LBT Beteiligungsgesellschaft, Germany, representing the Max- Planck Society, the Astrophysical Institute Potsdam, and Heidelberg University; The Ohio State University, and The Research Corporation, on behalf of The University of Notre Dame, University of Minnesota and University of Virginia.

\section{REFERENCES}

[1] Hill, J. M., Green, R. F., Ashby, D. S., Brynnel, J. G., Cushing, N. J., Little, J. K., Slagle, J. H., and Wagner, R. M., "The Large Binocular Telescope," 84441A, International Society for Optics and Photonics (sep 2012).

[2] Hinz, P., Bailey, V. P., Defrère, D., Downey, E., Esposito, S., Hill, J., Hoffmann, W. F., Leisenring, J., Montoya, M., McMahon, T., Puglisi, A., Skemer, A., Skrutskie, M., Vaitheeswaran, V., and Vaz, A., "Commissioning the LBTI for use as a nulling interferometer and coherent imager," 91460T-91460T-10 (2014).

[3] Skrutskie, M. F., Jones, T., Hinz, P., Garnavich, P., Wilson, J., Nelson, M., Solheid, E., Durney, O., Hoffmann, W., Vaitheeswaran, V., McMahon, T., Leisenring, J., and Wong, A., "The Large Binocular Telescope mid-infrared camera (LMIRcam): final design and status," 77353H, International Society for Optics and Photonics (jul 2010).

[4] "Instrument Neutral Distributed Interface." https://en.wikipedia.org/wiki/Instrument_Neutral_ Distributed_Interface. (Accessed: September 2015).

[5] Esposito, S., Riccardi, A., Fini, L., Puglisi, A. T., Pinna, E., Xompero, M., Briguglio, R., Quirós-Pacheco, F., Stefanini, P., Guerra, J. C., Busoni, L., Tozzi, A., Pieralli, F., Agapito, G., Brusa-Zappellini, G., Demers, R., Brynnel, J., Arcidiacono, C., and Salinari, P., "First light AO (FLAO) system for LBT: final integration, acceptance test in Europe, and preliminary on-sky commissioning results," 773609, International Society for Optics and Photonics (jul 2010).

[6] Esposito, S., Riccardi, A., Pinna, E., Puglisi, A. T., Quirós-Pacheco, F., Arcidiacono, C., Xompero, M., Briguglio, R., Busoni, L., Fini, L., Argomedo, J., Gherardi, A., Agapito, G., Brusa, G., Miller, D. L., Guerra Ramon, J. C., Boutsia, K., and Stefanini, P., "Natural guide star adaptive optics systems at LBT: FLAO commissioning and science operations status," 84470U-84470U-11, International Society for Optics and Photonics (sep 2012). 
[7] Defrère, D., Hinz, P., Downey, E., Ashby, D., Bailey, V., Brusa, G., Christou, J., Danchi, W. C., Grenz, P., Hill, J. M., Hoffmann, W. F., Leisenring, J., Lozi, J., McMahon, T., Mennesson, B., Millan-Gabet, R., Montoya, M., Powell, K., Skemer, A., Vaitheeswaran, V., Vaz, A., and Veillet, C., "Co-phasing the Large Binocular Telescope: status and performance of LBTI/PHASECam," 914609, International Society for Optics and Photonics (jul 2014).

[8] Rodigas, T. J., Debes, J. H., Hinz, P. M., Mamajek, E. E., Pecaut, M. J., Currie, T., Bailey, V., Defrere, D., De Rosa, R. J., Hill, J. M., Leisenring, J., Schneider, G., Skemer, A. J., Skrutskie, M., Vaitheeswaran, V., and Ward-Duong, K., "C:," The Astrophysical Journal 78321(12pp) (2014).

[9] Maire, A.-L., Skemer, A. J., Hinz, P. M., Desidera, S., Esposito, S., Gratton, R., Marzari, F., Skrutskie, M. F., Biller, B. A., Defrère, D., Bailey, V. P., Leisenring, J. M., Apai, D., Bonnefoy, M., Brandner, W., Buenzli, E., Claudi, R. U., Close, L. M., Crepp, J. R., De Rosa, R. J., Eisner, J. A., Fortney, J. J., Henning, T., Hofmann, K.-H., Kopytova, T. G., Males, J. R., Mesa, D., Morzinski, K. M., Oza, A., Patience, J., Pinna, E., Rajan, A., Schertl, D., Schlieder, J. E., Su, K. Y. L., Vaz, A., Ward-Duong, K., Weigelt, G., and Woodward, C. E., "The LEECH Exoplanet Imaging Survey. Further constraints on the planet architecture of the HR 8799 system," A $6 A$ 576(576), 133-133 (2015).

[10] Defrère, D., Hinz, P. M., Mennesson, B., Hoffmann, W. F., Millan-Gabet, R., Skemer, A. J., Bailey, V., Danchi, W. C., Downey, E. C., Durney, O., Grenz, P., Hill, J. M., Mcmahon, T. J., Montoya, M., Spalding, E., Vaz, A., Absil, O., Arbo, P., Bailey, H., Brusa, G., Bryden, G., Esposito, S., Gaspar, A., Haniff, C. A., Kennedy, G. M., Leisenring, J. M., Marion, L., Nowak, M., Pinna, E., Powell, K., Puglisi, A., Rieke, G., Roberge, A., Serabyn, E., Sosa, R., Stapeldfeldt, K., Su, K., Weinberger, A. J., and Wyatt, M. C., "NULLING DATA REDUCTION AND ON-SKY PERFORMANCE OF THE LARGE BINOCULAR TELESCOPE INTERFEROMETER," The Astrophysical Journal $\mathbf{8 2 4}$ (2016).

[11] Conrad, A., De Kleer, K., Leisenring, J., Camera, A. L., Arcidiacono, C., Bertero, M., Boccacci, P., Defrère, D., De Pater, I., Hinz, P., Hofmann, K.-H., Kürster, M., Rathbun, J., Schertl, D., Skemer, A., Skrutskie, M., Spencer, J., Veillet, C., Weigelt, G., and Woodward, C. E., "SPATIALLY RESOLVED M-BAND EMISSION FROM IO'S LOKI PATERAFIZEAU IMAGING AT THE $22.8 \mathrm{~m}$ LBT," The Astronomical Journal 149 (2015).

[12] Sallum, S., Follette, K., Eisner, J. A., Close, L., Hinz, P., Kratter, K., Males, J., Skemer, A., Tuthill, P., Bailey, V., Defrère, D., Morzinski, K., Rodigas, T., Spalding, E., Vaz, A., and Weinberger, A. J., "Accreting protoplanets in the LkCa 15 transition disk," Nature 527 (2015).

[13] Skemer, A. J., Hinz, P., Montoya, M., Skrutskie, M. F., Leisenring, J., Durney, O., Woodward, C. E., Wilson, J., Nelson, M., Bailey, V., Defrere, D., and Stone, J., "First light with ALES: A 2-5 micron adaptive optics Integral Field Spectrograph for the LBT," 96051D, International Society for Optics and Photonics (sep 2015).

[14] Skemer, A. J., Marley, M. S., Hinz, P. M., Morzinski, K. M., Skrutskie, M. F., Leisenring, J. M., Close, L. M., Saumon, D., Bailey, V. P., Briguglio, R., Defrere, D., Esposito, S., Follette, K. B., Hill, J. M., Males, J. R., Puglisi, A., Rodigas, T. J., and Xompero, M., "C:," The Astrophysical Journal 792(13pp) (2014).

[15] Matthews, C. T., Crepp, J. R., Skemer, A., Hinz, P. M., Gianninas, A., Kilic, M., Skrutskie, M., Bailey, V. P., Defrere, D., Leisenring, J., Esposito, S., and Puglisi, A., "MID-INFRARED HIGH-CONTRAST IMAGING OF HD 114174 B: AN APPARENT AGE DISCREPANCY IN A " SIRIUS-LIKE " BINARY SYSTEM," The Astrophysical Journal Letters 783 (2014).

[16] Bonnefoy, M., Currie, T., Marleau, G.-D., Schlieder, J. E., Wisniewski, J., Carson, J., Covey, K. R., Henning, T., Biller, B., Hinz, P., Klahr, H., Boyer, A. N. M., Zimmerman, N., Janson, M., Mcelwain, M., Mordasini, C., Skemer, A., Bailey, V., Defrère, D., and Thalmann, C., "Characterization of the gaseous companion $\kappa$ Andromedae b New Keck and LBTI high-contrast observations," A $\mathscr{Y} A 562$ (2014).

[17] Def Ere, D., Hinz, P. M., Skemer, A. J., Kennedy, G. M., Bailey, V. P., Hoffmann, W. F., Mennesson, B., Millan-Gabet, R., Danchi, W. C., Absil, O., Arbo, P., Beichman, C., Brusa, G., Bryden, G., Downey, E. C., Durney, O., Esposito, S., Gaspar, A., Grenz, P., Haniff, C., Hill, J. M., Lebreton, J., Leisenring, J. M., Males, J. R., Marion, L., Mcmahon, T. J., Montoya, M., Morzinski, K. M., Pinna, E., Puglisi, A., Rieke, G., Roberge, A., Serabyn, E., Sosa, R., Stapeldfeldt, K., Su, K., Vaitheeswaran, V., Vaz, A., Weinberger, A. J., and Wyatt, M. C., "FIRST-LIGHT LBT NULLING INTERFEROMETRIC OBSERVATIONS: WARM 
EXOZODIACAL DUST RESOLVED WITHIN A FEW AU OF $\eta$ Crv," The Astrophysical Journal 799, 42-9 (2015).

[18] Kennedy, G. M., Wyatt, M. C., Bailey, V., Bryden, G., Danchi, W. C., Ere, D., Haniff, C., Hinz, P. M., Lebreton, J., Mennesson, B., Millan-Gabet, R., Morales, F., Pani, O., Rieke, G. H., Roberge, A., Serabyn, E., Shannon, A., Skemer, A. J., Stapelfeldt, K. R., Su, K. Y. L., and Weinberger, A. J., "EXO-ZODI MODELING FOR THE LARGE BINOCULAR TELESCOPE INTERFEROMETER," The Astrophysical Journal Supplement Series 21623(13pp) (2015).

[19] Weinberger, A. J., Bryden, G., Kennedy, G. M., Roberge, A., Ere, D., Hinz, P. M., Millan-Gabet, R., Rieke, G., Bailey, V. P., Danchi, W. C., Haniff, C., Mennesson, B., Serabyn, E., Skemer, A. J., Stapelfeldt, K. R., and Wyatt, M. C., "TARGET SELECTION FOR THE LBTI EXOZODI KEY SCIENCE PROGRAM," The Astrophysical Journal Supplement Series 216(8pp) (2015). 